\title{
Blastoschizomyces capitatus pulmonary infections in immunocompetent patients: case report, case series and literature review
}

\author{
M. B. TANABE ${ }^{1 *}$ AND S. A. PATEL ${ }^{1,2}$ \\ ${ }^{1}$ Department of Internal Medicine, John H. Stroger, Jr. Hospital of Cook County, Chicago, IL, USA \\ ${ }^{2}$ Division of Hospital Medicine, Department of Medicine, John H. Stroger, Jr. Hospital of Cook County, \\ Chicago, IL, USA
}

Received 13 August 2017; Final revision 2 November 2017; Accepted 4 November 2017; first published online 4 December 2017

\section{SUMMARY}

Blastoschizomyces capitatus is an uncommon opportunistic yeast associated with infections in neutropaenic patients secondary to haematological malignancies, with a special predilection for the lungs. Globalisation and population migration impact on the epidemiology of infection with this organism but its effect on the immunocompetent population has rarely been described. We present here a case report, an overview of 11 other cases published between 2000 and 2016, and a comprehensive literature review of Blastoschizomyces pneumonia in the nonimmunocompromised. The median age at diagnosis was 68 years (range $40-86$ years) and more than half the cases reported a positive history of either current or past tobacco smoking. Six cases had either clinical or radiological evidence of chronic obstructive pulmonary disease and three had a history of prior treated tuberculosis. Fluconazole and itraconazole, alone or in combination, was the most utilised treatment. We conclude that unlike most other invasive yeast species, $B$. capitatus poses an infectious risk for immunocompetent patients, usually of middle to older age with risk factors for distorted lung architecture. Further research is warranted into the pathophysiology of Blastoschizomyces infections in the immunocompetent, including standardised treatment options.

Key words: Blastoschizomyces, fungal pneumonia, Geotrichum capitatum, immunocompetent, Saprochaete capitata, Trichosporon capitatum, yeast.

\section{INTRODUCTION}

Blastoschizomyces capitatus (a.k.a. Magnusiomyces capitatus, Trichosporon capitatum, Geotrichum capitatum and Saprochaete capitata) is a yeast species known to cause disseminated opportunistic infections - particularly in neutropaenic patients with haematologic

\footnotetext{
* Author for correspondence: M. B. Tanabe, John H. Stroger, Jr. Hospital of Cook County, 1901 West Harrison, 15th Floor (Administration Building), Chicago, IL 60612, USA. (Email: mtanabe@cookcountyhhs.org)
}

malignancies. Infections can range in severity, affecting the lungs, liver, brain, spleen, kidney and endocardium [1]. Its effect, however, in immunocompetent patients is rarely reported in the literature.

We describe here a case of an otherwise healthy elderly female from Egypt with past medical history of questionable chronic obstructive pulmonary disease (COPD) diagnosed with B. capitatus pneumonia. Subsequently, we carried out a comprehensive literature search on all reported cases worldwide of such infections in immunocompetent patients since 2000 and our findings are presented as a case series. 


\section{Case report}

An 86 year-old female who was visiting family in the United States (USA) from Egypt presented to the emergency department with shortness of breath, productive cough and fatigue. She had been in the USA for 2 months prior to presentation. She had a selfreported history of asthma, diagnosed in her late $60 \mathrm{~s}$ and was not taking any medications apart from albuterol MDI as needed. She denied any history of tuberculosis (TB), fever or chills, had never smoked and did not have any history of significant exposure to smoke, vapours, dusts or fumes.

On arrival at the emergency room, her vital signs were: blood pressure $130 / 48 \mathrm{mmHg}$, heart rate 110 beats per minute, respiratory rate of 22 and oxygen saturation of $100 \%$ on $4 \mathrm{~L}$ of nasal cannula. She was afebrile. Physical examination revealed an acutely ill-appearing thin female with laboured breathing, scattered wheezes and decreased breath sounds on the right side along with bilateral basilar crackles. The remainder of her systemic examination was unremarkable. Initial laboratory tests revealed a leucocytosis of $22.6 \mathrm{cel} 11 / \mathrm{mm}^{3}$ with a neutrophil predominance and a haemoglobin of $11.5 \mathrm{mg} / \mathrm{dl}$. All other laboratory examinations, including HIV screening, were within normal reference ranges.

A chest X-ray showed mild obstructive airway changes and a chest computed tomography (CT) was performed to rule out pulmonary embolism (PE) (Fig. 1). No PE was noted, however imaging demonstrated ill-defined opacities, 'tree-in-bud' nodules in bilateral lungs, bronchiectatic change and non-specific mediastinal lymphadenopathy. A diagnosis of a multilobar pneumonia was made on the basis of imaging results and clinical presentation.

The patient was started on albuterol and ipratropium nebulisers along with systemic corticosteroids for presumed superimposed asthma exacerbation. She was also initiated on ceftriaxone and azithromycin for community-acquired pneumonia. Given the radiographic findings and her country of origin, TB was considered but ruled out following three negative acid-fast bacilli smears. Histoplasma capsulatum and Legionella pneumophila antigen in urine were negative as well as blood cultures.

A sputum sample obtained on day 2 of admission showed many yeast cells with pseudohyphae by microscopy and culture by standard methods revealed two different yeast-like colony morphologies. By reference to the MicroScan yeast database, one of the

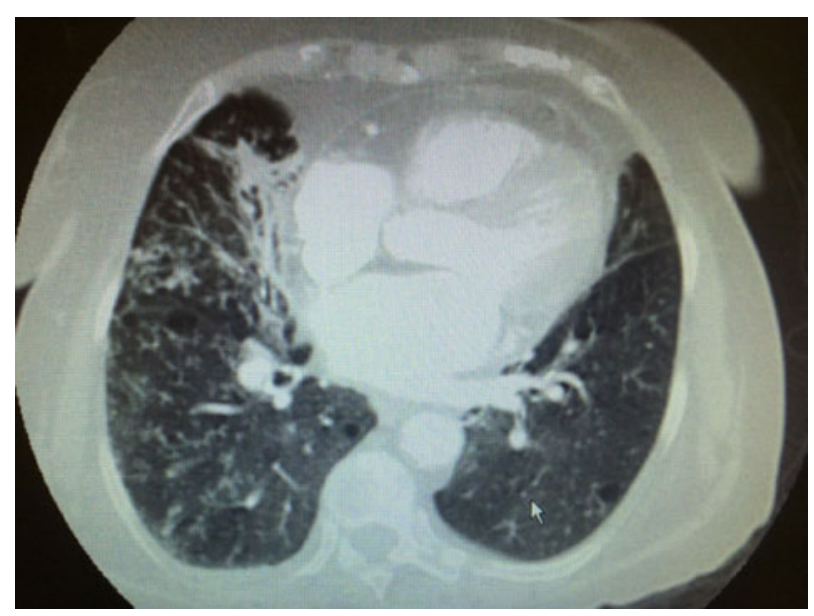

Fig. 1. Computerised tomography (CT) of the chest with contrast of an 86-year-old female from Egypt with past medical history of asthma. CT angiogram of the chest. Ill-defined opacities in the right middle lobe and peripheral right upper lobe with centrilobular nodules with three in bud appearance in the bilateral lungs, right more than left. Mildly enlarged lymph nodes within the mediastinum.

colony morphologies was consistent with $B$. capitatus, showing true hyphae, pseudohyphae and many annelloconidia as well as annellides forming along the hyphae and at the end of the branches; the other colonies were consistent with Candida albicans, showing blastospores, pseudohyphae and terminal chlamydospores. As Candida pneumonia is rare, even in immunocompromised patients, this isolate was considered to be a contaminant [2]. Antifungal susceptibility testing was not done.

On day 4 post-admission, the patient was noted to have increasing oxygen requirements, worsening tachypnoea, and infiltrates on repeat chest radiography despite treatment. She was transferred to the intensive care unit and started on non-invasive ventilator support along with antibiotic coverage with vancomycin and ceftazidime. On day 6, her clinical status improved sufficiently to be transferred back to the general medicine floor but despite continued treatment, recovery was not as rapid as expected for asthma complicated by simple bacterial community-acquired pneumonia. On day 12 of admission, a repeat dedicated CT chest (non-PE protocol) without contrast was done and showed minimal change, although there was marginal improvement in oxygen saturation levels to $90-92 \%$ on room air in a sedentary state. The patient was discharged on day 13 with oral amoxicillin-clavulanate to complete 14 days of antibiotic treatment without any oxygen requirements as she did not fulfil the criteria for home oxygen use at the time. 
Three days after discharge, a routine post-hospital clinic visit revealed no improvement in oxygen saturations, and on specialist consultative review, it was decided to initiate treatment with itraconazole for Blastoschizomyces infection. As she was returning to Egypt the following week, she was advised to follow-up her treatment there. Contact with the patient and her family approximately 1 year later revealed that she was doing well after completing 4 months of antifungal therapy, and had not received any other new medications or therapy. The family was unaware of any further imaging or microbiology testing done in Egypt.

\section{Case series}

A comprehensive literature search was performed via major medical databases (PUBMED, Cochrane) for case reports of immunocompetent patients with infection secondary to B. capitatum. The search strategy included the following: immunocompetent (B. capitatum or T. capitatum or G. capitatum or S. capitata or $M$. capitatum). Cases of such infections in immunocompromised patients were also reviewed for baseline reference. Exclusion criteria included patients $<18$ years old, infections attributed to malignancies or immunosuppression caused by any chemotherapy, uncontrolled diabetes (glycated haemoglobin $>8$ ), high steroid doses or AIDS/HIV. Inclusion criteria included patients $>18$ years old with diagnosis of Blastoschizomyces spp. via sputum \pm bronchoalveolar lavage (BAL) culture or endotracheal culture refractory improvement with appropriate antibiotic therapy, and improvement with antimycotic treatment.

Eleven cases were identified worldwide between 1996 and 2016, and patient demographics and background information are shown in Tables 1 and 2 . Cases were predominantly male with a median age at diagnosis of 68 years (range 40-86 years), and more than half reported a positive history of either current or past tobacco smoking; one case was associated with chewing tobacco, which was considered as a risk factor for infection. Six cases had either clinical or radiological evidence of COPD. Three cases had a history of prior treated TB but only one case had positive Mycobacterium avium-intracellulare cultures, however given improvement despite lack of treatment for such, it was considered not to be a factor for current clinical presentation. Five cases were reported from India [3-6], three from the USA including the case report above [7, 8], two from Nepal [9]
Table 1. Demographics and characteristics of Blastoschizomyces capitatus in immunocompetent cases reported between 1996 and 2016

\begin{tabular}{ll}
\hline \hline Background data & Total cases $(n=11)$ \\
\hline Age, years, median (range) & $68(40-86)$ \\
Male, sex, $n(\%)$ & $9(81 \cdot 8 \%)$ \\
Smoking history, $n(\%)$ & $7(63 \cdot 3 \%)$ \\
Other risk factors for lung disease & \\
Tuberculosis, $n(\%)$ & $3(27 \cdot 3 \%)$ \\
COPD, $n(\%)$ & $6(54 \cdot 5 \%)$ \\
MAC infection, $n(\%)$ & $1(9 \cdot 1 \%)$ \\
Silicosis, ILD, smoke exposure & 0 \\
Radiological evidence & \\
Consolidation, $n(\%)$ & $3(27 \cdot 2 \%)$ \\
Patchy infiltrates, $n(\%)$ & $4(36 \cdot 4 \%)$ \\
Tree in bud, BQE, $n(\%)$ & $1(9 \cdot 1 \%)$ \\
Fibrotic scarring, $n(\%)$ & $1(9 \cdot 1 \%)$ \\
Unknown, $n(\%)$ & $2(18 \cdot 1 \%)$ \\
\hline \hline
\end{tabular}

BQE, bronchiectasis; COPD, chronic obstructive pulmonary disease; ILD, interstitial lung disease; MAC, Mycobacterium avium complex.

and the other from Serbia [10]. Most diagnoses were made by sputum culture, and in only two patients, the pathogen was identified in BAL and sputum concurrently. Four patients in the series had a history of COPD [4, 5, 9]; two of them were on steroids, one was treated successfully with 2 weeks of fluconazole and another was initially treated with fluconazole before death [9]. Three cases had poor outcome which was attributed to no or inadequate treatment, and fluconazole (with or without combination therapy) was the most widely used antifungal (five cases) followed by itraconazole $(n=3)$; one reported case was successfully treated with voriconazole.

Of note, there were a few cases reported that were not included in our table due to the presence of exclusion factors. One described a 60-year-old immunocompetent critically ill male who became neutropaenic due to respiratory syncytial virus and Pseudomonas aeruginosa superinfection, and who grew Blastoschizomyces spp. in blood cultures [11]. It could not be ascertained whether the Blastoschizomyces infection was a contributing factor to the acute illness or a result of the subsequent neutropaenia. A second excluded case, a pleural abscess without pneumonia secondary to $M$. capitatus and possibly Aspergillus spp., was reported in a patient with a history of metastatic cancer, which could have inferred another degree of immunosuppression [12]. A further seven excluded cases of invasive and non-invasive pulmonary infections secondary to 
Table 2. Case reports worldwide of Blastoschizomyces capitatus infections in immunocompetent patients in the past 20 years

\begin{tabular}{|c|c|c|c|c|}
\hline Year & Location & Age gender $(\mathrm{M} / \mathrm{F})$ & Sample & Treatment and follow-up \\
\hline 2004 & FL, USA & 40-year-old non-smoker $\mathrm{M}$ with PNA & BAL sample & $\begin{array}{l}\text { Fluconazole and } \\
\text { amphotericin B } \\
\text { Good outcome [7] }\end{array}$ \\
\hline 2008 & Serbia & 46-year-old M smoker with PNA & Sputum and BAL & $\begin{array}{l}\text { Unsure } \\
\text { Deceased [10] }\end{array}$ \\
\hline 2011 & Karnataka, India & 58-year-old M with COPD and PNA & Sputum & $\begin{array}{l}\text { Amikacin } \\
\text { Deceased [4] }\end{array}$ \\
\hline 2013 & $\begin{array}{l}\text { Philadelphia, PA, } \\
\text { USA }\end{array}$ & $\begin{array}{l}\text { 64-year-old } \mathrm{M} \text { with a history of tobacco and } \\
\text { alcohol abuse }\end{array}$ & BAL & $\begin{array}{l}\text { Voriconazole } \\
\text { Good outcome [8] }\end{array}$ \\
\hline \multirow[t]{2}{*}{2014} & $\begin{array}{l}\text { Central Chennai, } \\
\text { India }\end{array}$ & 52-year-old $\mathrm{M}$ with TB and PNA & Sputum & $\begin{array}{l}\text { Itraconazole } \\
\text { Good outcome [5] }\end{array}$ \\
\hline & & 68-year-old M with COPD and PNA & Sputum & $\begin{array}{l}\text { Itraconazole } \\
\text { Good outcome [5] }\end{array}$ \\
\hline 2015 & Uttar Pradesh, India & 75-year-old M with PNA in old TB & Sputum & $\begin{array}{l}\text { Fluconazole } \\
\text { Good outcome [6] }\end{array}$ \\
\hline \multirow[t]{2}{*}{2015} & Pokhara, Nepal & 82-year-old M with COPD & $\begin{array}{l}\text { Sputum, ET } \\
\text { aspirate }\end{array}$ & $\begin{array}{l}\text { Fluconazole } \\
\text { Good outcome[9] }\end{array}$ \\
\hline & & 80-year-old M with COPD & Sputum, BAL & $\begin{array}{l}\text { Fluconazole } \\
\text { Deceased[9] }\end{array}$ \\
\hline 2015 & Chicago, IL, USA & 86-year-old F with PNA in asthma & BAL sample & $\begin{array}{l}\text { Itraconazole } \\
\text { Good outcome [This } \\
\text { report] }\end{array}$ \\
\hline
\end{tabular}

BAL, bronchoalveolar lavage; COPD, chronic obstructive pulmonary disease; ET, endotracheal; F, female; M, male; PNA, pneumonia; TB, tuberculosis.

M. capitatus in non-neutropaenic patients were reported from Nepal [9]. This series included one with invasive fungal infection in a patient with history of diabetes; another with cutaneous infection, and a third in a teenager with community-acquired pneumonia where $M$. capitatus was determined to be a contaminant due to clinical improvement without targeted therapy. Other cases in this series were a diabetic with pneumonia from whom $M$. capitatus was isolated at post-mortem, and another patient with multiple confounders (urinary tract infection, aspiration pneumonitis and sepsis) who grew the yeast from an endotracheal aspirate.

\section{DISCUSSION AND LITERATURE REVIEW}

Invasive and life-threatening fungal infections are more common in the immunocompromised patient and the majority of these are caused by Aspergillus spp. and Candida spp. [1, 13]. In the past decade, there has been an increase in infections secondary to uncommon opportunistic fungi. Non-Candida species account for between $1 \%$ and $5 \%$ of all disseminated yeast infections [14], and are significant contributors to the high mortality rates in about two-thirds of patients with end-stage cancer [15]. B. capitatus is a frequent constituent of the normal skin, gastrointestinal and upper respiratory tract flora and is more frequently reported as causing invasive infections in neutropaenic patients usually with haematologic malignancies [14-17]. Their clinical presentation is similar to that of the more common fungal infections. Over $90 \%$ of all Blastoschizomyces cases in immunocompromised patients have been reported from Europe, with significant numbers from Italy, Spain or France [14, 18]. A large survey of almost 3000 cases of fungaemia in a tertiary cancer centre in Houston, USA, found that $3 \cdot 1 \%$ were secondary infections due to non-Candida spp. Of these, $5 \%$ corresponded to $G$. capitatum, which was associated with a high mortality overall [19]. In another series of 88 cases of Blastoschizomyces infection, all had an underlying malignancy with acute myeloid leukaemia being the most common [20]. A positive blood culture with Blastoschizomyces was seen in $77 \cdot 3 \%$ of cases and $46 \%$ had disseminated infection reaching almost every organ, causing meningitis, osteomyelitis, 
endocarditis, hepatitis, gastroenteritis, nephritis and onychomycosis. Localised infection was seen most commonly in lung parenchyma (19.3\%). The organism has also been isolated from wood and soil, as well as catheters, intravenous fluids and environmental samples [21]. A nosocomial outbreak in a haematological unit was associated with milk contaminated by yeasts [22].

Blastoschizomyces spp. is now recognised as an emerging opportunistic pathogen, but was not considered pathogenic for humans until approximately 30 years ago by Salkin et al. [23]. Its effect in patients without previous immunosuppressive risk factors is not well understood but several cases have been reported around the world from immunocompetent patients with pneumonia (Table 2) secondary to Blastoschizomyces. It frequently precedes a clinical course of a lack of improvement with inappropriate antibiotic therapy. Common manifestations are cough with expectoration, chest pain, spontaneous pneumothorax and pulmonary infiltrates or consolidations often making it difficult to differentiate from other more common bacterial, viral or fungal infections [24].

Diagnosis of the infection relies solely on the identification of the organisms in sterile fluids or tissues. Blastoschizomyces is a non-fermentative, nonencapsulated, urease-negative ascomycetous yeast and is identified by lactophenol cotton blue preparations and Gram stain, with distinguishing microscopic characteristics such as septated hyphae and anneloconidia. The group grow on blood agar and Sabouraud Dextrose Agar as white to cream coloured colonies, and can grow at $45^{\circ} \mathrm{C}$ in the presence of cyclohexamide, but is unable to use potassium nitrate as a sole source of nitrogen or hydrolyse urea, and assimilates glucose and galactose [24-26].

This literature review and case series reports point to a new immunocompetent host for Blastoschizomyces infection, previously described as 'apparently immunocompetent' [4], predisposing them to fatal fungal infections. The yeast tends to infect most commonly the lungs particularly where there is distortion of the lung architecture with COPD, previous tobacco use and TB being the most common risk factors reported. Chauhan et al. [6] considered that pulmonary mycoses occur in non-neutropaenic patients who are either solid organ transplant recipients or have underlying chronic lung pathology. This might be explained by initial respiratory colonisation with the organism combined with environmental conditions and decreased lung protection barriers secondary to destruction of normal lung parenchyma. A healthy lung usually offers many lines for defence against fungal colonisation which although common, rarely progresses to infection. The upper respiratory epithelia act as a physical barrier to inhaled spores and deeper infection is combatted by phagocytosis by alveolar macrophages [27]. Natural killers and neutrophils form part of the second line of defence through cytokines and immunomodulators triggered by the fungus in its hyphal phase [28]. Yeast infections, such as Candida spp., are usually seen in those with an impaired T-cell response [29].

Most case reports of Blastoschizomyces infection in the immunocompetent originate from India, but it is recognised as an emergent infectious disease in haematological patients in Mediterranean areas, particularly in summer time which is indicative that climate plays a role in the epidemiology of infections at least in Europe. This is supported by the fact that most cases occur in locations below latitude $44^{\circ} \mathrm{N}$, which corresponds to hot and dry summers with wet relatively mild winters. Nevertheless, five cases were reported in central Switzerland in 2012 [30]. Given the current global trends in warming of climates and movements of populations across the world, it is highly possible that the frequency of Blastoschizomyces infections will increase and with a wider geographical distribution [31]; as was the case of our patient reported above who was originally from Egypt but had a history of travelling to different parts of the world before being diagnosed in the USA.

Blastoschizomyces infections appear to be seen most commonly in middle-aged males, although there are no published data on gender or age preference. As for several other infections, the elderly population are usually at higher risk given more comorbidities and compromised immune function.

The outcome of the infection with rare invasive yeasts relies largely on the patient's immune system [24]. It has been observed that for patients with profound neutropaenia, mortality ranges between $50 \%$ and $90 \%$, and survival is closely linked to recovery of the neutrophil count [14] and good immune function is necessary for the clearance of non-Candida spp. from the bloodstream despite appropriate antifungal treatment [15]. The azole compounds have played a major role in the treatment of yeast infections, and fluconazole with or without the combination of amphotericin B is widely acknowledged to be the most effective for treatment of Blastoschizomyces infections with minimum inhibitory concentrations 
(MIC) ranging from $0 \cdot 25-8 \mu \mathrm{g} / \mathrm{ml}$ for fluconazole and $0 \cdot 03-0 \cdot 125 \mu \mathrm{g} / \mathrm{ml}$ for itraconazole and voriconazole $[32,33]$.

Currently, there are no established guidelines concerning the most appropriate antifungal agent for the treatment of Blastoschizomyces infections. B. capitatus has been reported as susceptible to amphotericin B with a MIC range of $0 \cdot 15-0.64 \mu \mathrm{g} / \mathrm{ml}$ and intermediate susceptible to 5-fluorocytosine (flucytosine) (MIC range 0.04 to $>100 \mu \mathrm{g} / \mathrm{ml}$ ) with fluconazole having four to eight times greater activity than amphotericin B [7]. Others have also found poor in vitro MIC activity for amphotericin B, but clinical improvement has been noted in some patients treated with amphotericin B and flucytosine [11,34]. Resistance to caspofungin has been reported [32], which might be due to acquired resistance owing to its wide use as part of empirical antifungal treatment in neutropaenic fevers.

Based on our case reports, a rapid clinical response is usually seen within 5-7 days of treatment with only two documenting unfavourable outcomes. In one case, the patient had not received an antifungal agent and the other patient was treated inappropriately with amikacin and had prior advanced disseminated disease [4]. We therefore suggest that the azole compounds and amphotericin B should be considered as first-line agents, although optimal duration of treatment would have to be based on clinical judgement in the absence of definitive information.

Our case study had some limitations. First, the MicroScan database used in our hospital for fungal identification was suboptimal given the evolving taxonomy of this large fungal group, and has been largely supplanted by PCR sequencing of the internal transcribed spacer, and/or matrix-assisted laser desorption ionisation-time of flight mass spectrometry analysis [35]. Second, antifungal susceptibility was not carried out on our isolate, and therapy was guided by reference to the literature.

In conclusion, Blastoschizomyces spp. are emerging opportunistic pathogens capable of causing disease in both immunocompromised, particularly in neutropaenia secondary to haematological malignancies, and immunocompetent individuals. The literature suggests that other risk factors such as chronic underlying lung pathology might contribute to the pathogenesis of pulmonary Blastoschizomyces spp. in immunocompetent hosts. Infection commonly occurs in middle-aged males, usually in Europe but is not limited to this area. Treatment is based on azoles, with fluconazole being the most widely used. It is important to create awareness of such pathogens which if not diagnosed in a timely manner, can lead to detrimental outcomes.

\section{ACKNOWLEDGEMENTS}

The authors thank Ijeoma Ezeife, MD, for her collaboration on the editing of the case report; Hemil Gonzalez, MD, for his collaboration on the interpretation of the microbiological specimen; and William Janda, $\mathrm{PhD}$, for his assistance in the interpretation of microbiologic data.

\section{DECLARATION OF INTEREST}

None.

\section{ETHICAL APPROVAL}

This article does not contain any studies with human participants performed by any of the authors.

\section{INFORMED CONSENT}

For case series, formal consent is not required. Informed consent was obtained from the individual of case report stated in this article.

\section{REFERENCES}

1. Groll AH, Walsh TJ. Uncommon opportunistic fungi: new nosocomial threats. European Journal of Clinical Microbiology and Infectious Diseases 2001; 7: 8-24.

2. Haron E, et al. Primary Candida pneumonia. Experience at a large cancer center and review of literature. Medicine (Baltimore) 1993; 72: 137-142.

3. Gill PK, Gill JS. Blastoschizomyces capitatus pneumonia in an immunocompetent female. Indian Journal of Tuberculosis 2011; 58: 88-89.

4. Sreeja S, et al. Blastoschizomyces capitatus pneumonia: a rare case. Indian Journal of Pathology and Microbiology 2011; 54: 846-847.

5. Dhevahi E, et al. Blastoschizomyces capitatus an rare opportunistic infection alerts the clinical correlation from a territory care hospital at Chennai. Journal of Dental and Medical Sciences 2014; 13: 59-62.

6. Chauhan S, et al. Pulmonary infection by Blastoschizomyces capitatus in an old tuberculosis patient. Journal of Clinical and Diagnostic Research 2015; 9: 1-2.

7. Wills TS, et al. Blastoschizomyces capitatus pneumonia in an immunocompetent male. Southern Medical Journal 2004; 97: 702-704.

8. Vahid B. Blastoschizomyces capitatus infection in an immunocompetent man. Journal of Respiratory Diseases 2007; 28: 82. 
9. Subramanya Supram H, et al. Emergence of Magnusiomyces capitatus infections in Western Nepal. Medical Mycology 2016; 54: 103-110.

10. Pesut D, et al. Bilateral pulmonary shadows and incapsulated mediastinal effusion in a patient with respiratory blastoschizomycosis. Pneumonologia 2008; 57: 88-91.

11. Miglietta F, et al. A rare case of Saprochaete capitata fungemia in a critical ill patient without hematologic and oncological disorders. Revista Espanola de Quimioterapia 2016; 29: 47-49.

12. Brunetti G, et al. Management and treatment of Magnusiomyces capitatum (Geotrichum capitatum) pleural infection in a non-neutropenic patient with posaconazole. A new therapeutic opportunity? The New Microbiologica 2016; 39: 307-309.

13. Pfaller MA, Diekema DJ, Merz WG. Infections caused by non-Candida, non-Cryptococcal yeasts. Clinical Mycology 2009; 2: 261.

14. Arendrup MC, et al. ESCMID and ECMM joint clinical guidelines for the diagnosis and management of rare invasive yeast infections. European Society of Clinical Microbiology and Infectious Diseases 2013; 20: 76-98.

15. Prodhan T, et al. Non Candida yeast fungemia in cancer patients: a retrospective analysis at a referral cancer center, 1999-2014. Infectious Diseases in Clinical Practice 2015; 23: 260-262.

16. Martino P, et al. Blastoschizomyces capitatus: an emerging cause of invasive fungal disease in leukemia patients. Reviews of Infectious Diseases 1990; 15: 570 582.

17. Malani AN, Kauffman CA. Changing epidemiology of rare mould infections: implications for therapy. Drugs 2007; 67: 1803-1812.

18. Fasciana T, et al. Genotyping and antifungal susceptibility of Dipodascus capitatus isolated in a neonatal intensive care unit of a Sicilian hospital. Advances in Experimental Medicine and Biology 2017; 973: 81-88.

19. Chitasombat MN, et al. Rare opportunistics (nonCandida, non-Cryptococcus) yeast bloodstream infections in patients with cancer. Journal of Infection 2012; 64: 68-75.

20. Girmenia C, et al. GIMEMA Infection Program. Invasive infections caused by Trichosporon species and Geotrichum capitatum in patients with hematological malignancies: a retrospective multicenter study from Italy and review of the literature. Journal of Clinical Microbiology 2005; 43: 1818-1828.

21. Mathews MS, Sudipta S. Blastoschizomyces capitatus infection after contamination of fluids for intravenous application. Mycoses 1998; 41: 427-428.
22. Gurgui M, et al. Nosocomial outbreak of Blastoschizomyces capitatus associated with contaminated milk in a haematological unit. Journal of Hospital Infection 2011; 78: 274-278.

23. Salkin IF, et al. Blastoschizomyces capitatus, a new combination. Mycotaxon 1986; 22: 375-380.

24. Bouza E, Munoz P. Invasive infections caused by Blastoschizomyces capitatus and Scedosporium spp. Clinical Microbiology and Infection 2004; 10: 76-85.

25. Pontieri E, et al. Genetic variability among Blastoschizomyces capitatus isolates from different clinical sources. International Journal of Immunopathology and Pharmacology 2005; 18: 531-539.

26. Freydiere AM, Guinet R, Boiron P. Yeast identification in the clinical microbiology laboratory: phenotypical methods. Medical Mycology 2001; 39: 9-33.

27. Boyton R, Openshaw PJ. Pulmonary defences to acute respiratory infections. British Medical Bulletin 2002; 61: 1-12.

28. Segal B. Aspergillosis. New England Journal of Medicine 2009; 360: 1870-1884.

29. Kullberg BK, Arendrup M. Invasive candidiasis. New England Journal of Medicine 2015; 373: 1445-1456.

30. Birrenbachm T, et al. Emergence of Blastoschizomyces capitatus yeast infections, Central Europe. Emerging Infectious Diseases 2012; 18: 98-101.

31. Garcia-Solache MD, Casadevall A. Global warming will bring new fungal diseases for mammals. MBio 2010; 1: $1-3$.

32. Nedret Koc A, et al. Molecular epidemiology and antifungal susceptibility of Saprochaete capitata (Blastoschizomyces capitatus) isolates causing nosocomial infection in Kayseri/Turkey. Infectious Diseases (London, England) 2016; 48: 596-603.

33. Sancak B, et al. Investigation of in vitro activities of fluconazole, itraconazole and voriconazole against clinical isolates of Blastoschizomyces capitatus (Geotrichum capitatum) by two different methods. Mikrobiyoloji Bulteni 2009; 43: 269-276.

34. Chittick P, et al. Case of fatal Blastoschizomyces capitatus infection occurring in a patient receiving empiric micofungin therapy. Antimicrobial Agents and Chemotherapy 2009; 53: 5306-5307.

35. Desnos-Ollivier M, et al. Misidentification of Saprochaete clavata as Magnusiomyces capitatus in clinical isolates: utility of internal transcribed spacer sequencing and matrix-assisted laser desorption ionization-time of flight mass spectrometry and importance of reliable databases. Journal of Clinical Microbiology 2014; 52: 2196-2198. 